\title{
Application of Iconport single incision sealing device in laparoscopic surgery.(Experience in 125 cases of appendectomy)
}

\section{Xiaojun Wang}

The Affiliated Hospital of Medical School of Ningbo University https://orcid.org/0000-0002-6264-8238 Haibo Jin ( $D$ 1977628782@qq.com )

\section{YongFeng Shuai}

The Affiliated Hospital of Medical School of Ningbo University

\section{YiZhong Zhang}

The Affiliated Hopital of Medical School of NingBo University

\section{Research article}

Keywords: Transumbilical; Self-made single port device ; Iconport; Laparoscopic appendectomy

Posted Date: September 7th, 2019

DOI: https://doi.org/10.21203/rs.2.14065/v1

License: (c) (i) This work is licensed under a Creative Commons Attribution 4.0 International License. Read Full License 


\section{Abstract}

Background To discuss the reliability and clinical value of Iconport, a Self-made single port device, which was applied for transumbilical single port laparoscopic appendectomy. Methods The incision was around $2.5 \mathrm{~cm}$ long with curved form around umbilicus. And the skin and each layer of the abdominal wall were cut in sequence. Then the silica gel sleeve of the self-made single-incision sealing device was folded, clamped by the forceps, and carefully inserted into the incision for sealing. The operation of laparoscopic appendectomy was accomplished by-use routine linetype laparoscopic instruments via Iconport. The specimen was discharged from peritoneal cavity through the single port device. Two layers of incision, linea alba abdominus and skin, were separately sutured by absorbable thread and each layer was sutured successively. Results Among the 125 patients, 119 were successfully performed single-port laparoscopic appendectomy, with the operation time ranging from 30 to $110 \mathrm{~min}(69.34 \pm 21.4 \mathrm{~min}$ on average). In 6 cases, auxiliary operating hole was added or laparotomy was performed. Total hospital stay was $5.0 \pm 1.8$ (2-14) days. 5 cases of incision infection. All patients were followed up for 1 to 6 months after surgery, with an average of 3 months. There were no complications such as bleeding, intestinal leakage, intestinal obstruction, and residual peritoneal effusion. Conclusion In single-incision laparoscopic surgery, the u-shaped incision around the umbilical margin of $2.5 \mathrm{~cm}$ for adult can not only meet the requirements of flexibility of instruments in the operation, but also meet the aesthetic requirements because the incision scar is hidden in the umbilical foramen or looks like the umbilical foramen after the operation. Iconport single-hole device, which has potential promotion value, can be used in relatively simple laparoscopic surgery alone or combined with a single puncture device for relatively complex laparoscopic surgery.

\section{Background}

Single-port laparoscopy has been a research hotspot in the field of minimally invasive surgery for recent 20 years $^{[1]}$. It has not been widely used yet for the need of special auxiliary instruments and the relative difficulty of operation. Different single-hole devices have different characteristics in terms of operational flexibility ${ }^{[2-11]}$. The author has been engaged in the research of a patent product of laparoscopic singlehole device since 2011 ${ }^{[12]}$. (Product name: Iconport, Chinese patent No. : 201110229309.4 US patent No. : US9,393,003B2)( Figure 1). The application experience of Iconport single-port device in laparoscopic appendectomy is briefly discussed based on 125 cases completed in the period from January 2016 to December 2018.

\section{Methods}

Patient selection

The data of this group were retrospectively studied. A total of 125 patients (77 males and 48 females) who underwent laparoscopic appendectomy through umbilicus were collected during the period of January 1, 2016 and December 31, 2018 in this treatment group, who were all diagnosed as appendicitis 
and appendiceal abscess after conservative treatment three months later. The average age was $38.2 \pm 14.8$ (12-69) years old and BMI was 23.2 $\pm 3.5(17.3-31.6)$. All the patients had right lower quadrant abdomen pain and located tenderness in the right lower quadrant abdomen. Some patients had fever but the temperature of all the patients did not exceed $38.5^{\circ} \mathrm{C}$. The course of disease was $10 \sim 120 \mathrm{~h}$, and the mean time was $28 \mathrm{~h}$. Preoperative ultrasonography or CT scan of the lower abdomen suggested hypoechoic mass in the right lower abdomen, exudation around ileocecal area or visible fecalith in the appendix cavity. And urinary calculi, ectopic pregnancy and other diseases were excluded. There were 114 cases with elevated WBC $\left(10 \times 10^{9} / \mathrm{L} \sim 23 \times 10^{9} / \mathrm{L}\right)$, and 11 cases with normal WBC. There were 18 cases of acute simple appendicitis, 5 cases of chronic appendicitis, 87 cases of acute suppurative appendicitis, 11 cases of acute gangrenous appendicitis, and 4 cases of periappendiceal abscess in this study according to postoperative clinical diagnosis. Case selection criteria: preoperative diagnosis of appendicitis was basically clear, stable vital signs, cardiopulmonary function can tolerate general anesthesia; Laparoscopic surgery was acceptable. Exclusion criteria: Patients with clinical or radiological suspicion of appendicular pathology were complicated by an abscess and/or local or diffused peritonitis. Patients with septic shock, patients with contraindications for laparoscopic surgery patients with contraindications for general anesthesia, the age below 12 years, pregnant patients, and patients who were unable to sign the informed consent form because of amental disorder were all excluded. Discharge standard: The temperature was below 37 degrees for more than 48 hours. Blood routine examination showed normal WBC and CRP was less than $40 \mathrm{mg} / \mathrm{L}$. No redness, swelling and exudation was found in the incision. Tolerance to oral diet, ambulation and absence of uncontrolled pain. All surgeries were performed after obtaining informed consent from the patients, and the study was approved by our Institutional Review Board.

Surgical techniques

U-shaped incision, about 2.5-3 cm long, was designed along the left annulus fold of umbilicus. Three towel pliers lifted the incision at both ends and the midpoint respectively, and the scalpel penetrated into the skin and subcutaneous tissue along the lateral wall of the umbilicus(Figure 2). The tissue was cut around the umbilicus to open the linea alba then open the peritoneum. Fold the bottom of the incision sealing sleeve and insert it into the incision. Pull it up so that the bottom surface of the incision sealing sleeve is tightly attached to the abdominal wall. Connect the pipe of the pneumoperitoneum to the intake pipe of the single-hole device to establish pneumoperitoneum with pneumoperitoneum pressure of 12$14 \mathrm{mmHg}$. In general, the lens enter the operating area through the central operating hole. The laparoscopic instruments can be used to complete the operation with the two operating holes adjacent to each other or the two operating holes on both sides of the lens. Generally, $10 \mathrm{~mm}$ lens and ordinary straight-rod laparoscopic surgical instruments are used to complete the operation, and the operation process of appendectomy is basically the same as that of conventional laparoscopic surgery(Figure 3 ). In special cases, laparoscopic instruments that can be bent are used to complete the operation. After the appendix is removed, the operating panel is removed and the specimen is taken out of the peritoneal cavity under the protection of the incision seal sleeve. If drainage is necessary, use the negative pressure 
drainage ball to draw out the body through the umbilical incision. The linea alba and peritoneum of the incision are closed by continuous suture with 2-0 absorbable suture. The incision should be thoroughly rinsed. Continuous intradermal suture with 4-0 absorbable suture is performed on the skin. The umbilicus is wrapped up under compression by small gauze strips.

\section{Data Collection}

The patients' age, sex, body mass index, operative time, bleeding volume, conversion, postoperative hospital stay, follow-up duration and complications were recorded. All of these patients were followed up by phone calls. The results are expressed as means \pm SD or numbers (percentages).

\section{Results}

There were 125 cases of acute and chronic appendicitis or appendiceal abscess after conservative treatment for three months of elective surgery in this study, and 119 cases of them successfully were completed single-port laparoscopic appendectomy with Iconport single incision sealing device . 1 case underwent the operation with additional auxiliary operation hole due to accidental hemorrhage of appendix artery. 1 case was diagnosed cecal diverticulum fecalstone incarceration during the operation ,which caused diverticulum suppurative inflammation, and intestinal repair was performed by adding auxiliary operation holes, 4 cases were severely wrapped around the appendix, and there was a lot of blood oozing during the operation, Among them, 3 cases added auxiliary operating hole to complete the operation and 1 case changed open operation. The operation time was 30 110 min, with an average of $69.34 \pm 21.4 \mathrm{~min}$. Total hospital stay was $5.0 \pm 1.8$ days, range(2-14) days. Wound infection was reported in 5 cases, and wound healing was gradually improved by strengthening wound management and prolonging antibiotic use time. Visual analogue scoring (VAS) was used to evaluate the pain scores of the incisions $1 d, 2 d$ and $3 d$ after the operation, and the scores of the non-infected incisions ranged from 1-3 points, with an average score of $1.8 \pm 0.7$ points. All patients were followed up for 1 to 6 months after surgery, with an average of 3 months. There were no complications such as bleeding, fecal leakage, intestinal obstruction, and residual peritoneal effusion, etc.

\section{Discussion}

Appendectomy is one of the most common operations in abdominal surgery. Most appendectomies are simple, and a few are complicated because of the condition. The transumbilical single port laparoscopic technique became the hotspot in the field of surgery had a history of 20 years, but it is popularized slowly, mainly because there is limited vision and adverse factors such as operating devices interfere with each other when the lens and the two operating instruments work together through the same small incision. Different laparoscopic single-hole devices have different characteristics in terms of operational flexibilit Since 2011. The author has developed a laparoscopic single-port device (Iconport), and tried it on more than 200 cases of tranumbilical single-port laparoscopic appendectomy. The data of this group are from the surgical practice of the same surgeon using the same device and similar methods after the learning 
period. In the past, most surgeons believed that the greatest benefit of single-hole surgery lay in the aesthetic incision, so the incision must be very small. The author thinks there is some prejudice in this view. In this study, the author used three cloth pliers to locate and pull the skin at the edge of the umbilicus foramen, and the scalpel was inserted subcutaneously to cut the skin, so that the incision line ran strictly on the skin fold around half of the umbilicus foramen(Figure 2), to ensure the aesthetic appearance of the surgical mark(Figure 4). In the author's opinion, the aesthetic degree of the incision mainly depends on the radius distance between the incision line and the central point of the umbilical foramen. The closer the u-shaped incision is to the umbilical hole, the more satisfied it is to hide the surgical mark. The length of the incision is not the main factor affecting the aesthetic quality. The aesthetic degree of incision is directly related to the original shape of umbilical foramen. The design of the umbilical incision is a u-shaped incision around the umbilicus, so that the incision mark can be "hidden in the umbilical foramen " as far as possible. In some patients, the umbilical foramen is small and the skin surrounding the umbilical foramen is flat and lacks the normal skin folds, which cannot be completely hidden by any kind of incision. The u-shaped incision made by the method described in the paper can make the postoperative incision mark "look like umbilical foramen ". In other words, the morphology of umbilical foramen after surgery may be slightly different from that of the patient before surgery, but the appearance of the surgical mark of u-shaped skin incision looks like the common morphology of umbilical foramen, thus hiding the surgical mark objectively. The overemphasis on smaller incisions makes all single-port laparoscopic procedures very difficult and artificially increases the risk of intraoperative procedures causing accidents. The author suggests that the lconport device should be used for single-port laparoscopic surgery with an incision of $2.5 \mathrm{~cm}$ in adults. In the process of singlehole laparoscopic surgery with a small incision of $2.5 \mathrm{~cm}$, two laparoscopic operating instruments can form an effective operational triangle. There are 4 operation holes on the operation panel of Iconport. The lens enters the surgical field through the middle hole. The center distance of the operating holes on both sides of the lens is $4 \mathrm{~cm}$ and which of the two operating holes in the adjacent position is $3 \mathrm{~cm}$. When the ends of the two instruments reach the same point in the abdominal cavity, the width of the instruments passing through the incision should be less than $2.5 \mathrm{~cm}$. Therefore, the $2.5 \mathrm{~cm}$ incision can ensure the flexible operation by surgical instruments. Using different operating holes in pairs in operation can solve the problem of unsatisfactory visual field exposure in the single operating hole scheme. Although an incision of $2.5 \mathrm{~cm}$ to $3 \mathrm{~cm}$ in the right lower quadrant of the abdomen can be used for some simple appendectomy, the same $2.5 \mathrm{~cm}$ incision around the umbilical border allows most appendectomies to be done with laparoscopic instruments, Moreover, the incision infection rate is low, the incision pain is light, and the incision mark is hidden in the umbilical foramen, which are the advantages of transumbilical single-port laparoscopic surgery ${ }^{[13]}$. In this study, such small sum of operations (6 cases) were completed by adding auxiliary operation holes or switching to open operation. The main reasons are as follows: 1 . Unclear anatomical relationship caused by inflammatory dense adhesion. 2. Increased brittleness of appendix, with the risk of rupture near the root of appendix. 3. Accidental bleeding and attempted hemostatic measures are not effective. 4. Misdiagnosis and accidental injury. In fact, in the process of "three-hole" laparoscopic surgery, most surgeons need to add auxiliary operating holes to change to "fourhole" or change to open surgery in these conditions. According to the author's experience, single-port 
laparoscopic appendectomy can be carried out smoothly if the appendix can be effectively pulled after preliminary separation and the appendix can withstand a certain pull strength. When severe appendicitis occurs and there is risk of rupture caused by traction, careful evaluation should be made to determine whether the operation can be successfully completed through gentle operation. Otherwise, auxiliary operation holes should be added in time. Once the appendix ruptures from the root, it was difficult to complete the operation through a single hole in most cases. When the position of retroperitoneum appendix was difficult to be exposed, the surgical field can be exposed with the help of small gauze strips and turnable laparoscopic surgical instruments. After turning the head of the instruments, intestines and mesentery can be effectively blocked to the medial side like the effect of retractor. In this study, the infection rate of incision was $4.8 \%(6 / 125)$. All the 5 incisions with early infection had the problem of inadequate irrigation before incision closure. The root reason why incision infection can be effectively controlled is that the removal of the operating panel during the operation and the removal of the specimen through the incision sealing sleeve completely avoid the risk of contact between the specimen and the incision. Compared with conventional three-hole laparoscopic surgery, the literature has different conclusions on whether the incision pain is increased or reduced ${ }^{[8-16]}$. VAS score of postoperative incision pain in this group was 1-3 points, and no patients needed to use analgesic drugs to treat incision pain. The author thinks the reason for the inscision mildly pain was related by the different incision methods. The data of this study were about the incision around the edge of umbilical foramen. The incision was relatively large, and the suture needle can enter into the incision to firmly suture the aponeurotic layer tissue. Because the umbilical incision surgery avoided the injury to the bilateral rectus abdominis, the postoperative events were less likely to cause incision pain due to the activities of daily life. The author believes that the probability of secondary incisional hernia of umbilical incision after umbilical singlehole laparoscopic surgery is decreased rather than increased, which is compared with that of three-hole laparoscopic puncture, because the large incision is conducive to suture tightly. In laparoscopic surgery, there is a common unreasonable phenomenon of "small hole operation and large hole specimen removed ", especially in laparoscopic gastrointestinal tumor surgery. Since the whole specimen needs to be removed, an incision of at least $3-4 \mathrm{~cm}$ is necessary before the specimen can be removed from the body. The existing plan was to complete the operation with laparoscope first, then enlarged one of the operating holes or make another small incision to remove specimens, and then reconstruct pneumoperitoneum to complete the subsequent operation process. In these cases, the incision needed for remove the specimens was not fully utilized in the whole operation process. The medical silicone seal sleeve for Iconport single-hole device to enter the incision has different specifications, including $1 \mathrm{~cm}$, $2 \mathrm{~cm}, 3 \mathrm{~cm}, 5 \mathrm{~cm}$ etc, depending on the incision diameter. The device is suitable for clinicians to choose according to the operation difficulty of specific surgery and whether there is a need to remove samples. $2 \mathrm{~cm}$ size is commonly used for general appendix surgery, while $1 \mathrm{~cm}$ size is optional for children. Obviously, the larger the incision is, the less difficulty the operation will be and the smaller the incision is, the more pretty the appearance of the operation mark is. For laparoscopic surgery with an incision is larger than $3 \mathrm{~cm}$ required for specimen remove, the umbilical incision should be made according to the requirements of specimen which should remove at the beginning of the operation, so as to achieve the 
goal of reducing and narrowing the laparoscopic puncture hole and to give consideration to the operating habits of surgeons without increasing the difficulty and risk of surgery.

\section{Conclusions}

The author thinks that in single-incision laparoscopic surgery, the u-shaped incision around the umbilical margin of $2.5 \mathrm{~cm}$ for adult can not only meet the requirements of flexibility of instruments in the operation, but also meet the aesthetic requirements because the incision scar is hidden in the umbilical foramen or looks like the umbilical foramen after the operation. Iconport single-hole device, which has potential promotion value, can be used in relatively simple laparoscopic surgery alone or combined with a single puncture device for relatively complex laparoscopic surgery.

\section{Abbreviations}

BMl,body mass index

\section{References}

1. Rispoli G,Armellino MF,Esposito C.One-trocar appendectomy. Surg Endosc. 2002;16:833-5.

2. Saber AA, Elgamal MH, Itawi EA, Single incision laparoscopic sleeve gastrectomy (SILS): a novel technique.Obes Surg. 2008;18:1338-42.

3. Andre Chow, Sanjay Purkayastha, and Paraskevas Paraskeva. Appendicectomy and Cholecystectomy Using Single-Incision Laparoscopic Surgery (SILS): The First UK Experience. Surgical Innovation Volume 16 Number 3 September 2009:211-217.

4. Daniel J. Ostlie. Single-site umbilical laparoscopic appendectomy. Seminars in Pediatric Surgery (2011) 20, 196-200.

5. Sathyaprasad C. Burjonrappa, Hrishikesh Nerkar. Teaching Single-Incision Laparoscopic Appendectomy in Pediatric Patients. JSLS (2012)16:619-622.

6. Ma Dolores Frutos, Jesus Abrisqueta, Juan Lujan, Randomized Prospective Study to Compare Laparoscopic Appendectomy Versus Umbilical Single-Incision Appendectomy. Annals of Surgery Volume 257, Number 3, March 2013:413-418.

7. Jonathan T Carter, Jennifer A Kaplan, Jason N Nguyen, A Prospective, Randomized Controlled Trial of Single-Incision Laparoscopic vs Conventional 3-Port Laparoscopic Appendectomy for Treatment of Acute Appendicitis. J Am Coll Surg 2014 218(5):951-959.

8. Stavros A. Antoniou, Oliver O. Koch, George A. Antoniou,. Meta-analysis of randomized trials on single-incision laparoscopic versus conventional laparoscopic appendectomy. The American Journal of Surgery (2014) 207, 613-622.

9. Rodrigo Nascimento PINHEIRO, Renato Costa SOUSA, Fernanda Mesquita de Brito CASTRO, Singleincision videolaparoscopic appendectomy with conventional video laparoscopy equipment.ABCD 
Arq Bras Cir Dig 2014;27(1):34-37.

10. Paul Buckley III,Hannah Vassaur,Sharon Monsivais, etal. Single-incision laparoscopic appendectomy versus traditional three-port laparoscopic appendectomy: an analysis of outcomes at a single institution. Surg Endosc (2014) 28:626-630.

11. Yongsheng Chen, Shuodong Wu, and Jing Kong. Transumbilical Single-Incision Laparoscopic Combined Cholecystectomy and Appendectomy: A Retrospective Comparative Study. Journal of laparoendoscopic \& advanced surgical techniques Volume 24, Number 10, 2014:702-706.

12. Wang XJ, Zhang YZH, Shuai YF, Application of Iconport in single-port laparoscopic appendectomy: a report of 30 cases. Chinese journal of minimally invasive surgery 2014(9):837-838.

13. Tezcaner T, Arer MI, Kidnap M, Long-term cosmetic results of single-incision vs. conventional laparoscopic appendectomy A prospective observational cohort study. Ann Ital Chir. 2018;89:448454.

- Yoshida M, Oshiro T,Fukuda T, et al. Single-incision laparoscopic surgery using the glove technique with improved maneuverability achieved through fulcrum creation via a simple ring technique. Asian J Endosc Surg.2019;1-3.

- Liao YT , Lai PS , Hou YZ, Is single-incision laparoscopic appendectomy suitable for complicated appendicitis? A comparative analysis with standard multiport laparoscopic appendectomy. Asian J Surg. 2019 May 23.

- Moriguchi T, Machigashira S,Sugita K,Kawano M, A Randomized Trial to Compare the Conventional Three-Port Laparoscopic Appendectomy Procedure to Single-Incision and One-Puncture Procedure That Was Safe and Feasible, Even for Surgeons in Training. J Laparoendosc Adv Surg Tech A. 2019 Mar;29(3):392-395.

\section{Declarations}

- Ethics approval and consent to participate This study has been reviewed by the research ethics committee of the Affiliated Hospital of Medical School of Ningbo University. Reference Number:2019KY0402. Written informed consent has been obtained from all participants in this study.

Consent for publication All information in this study was approved for publication by all participants.

- Availability of data and material The datasets used and/or analysed during the current study are available from the corresponding author on reasonable request.

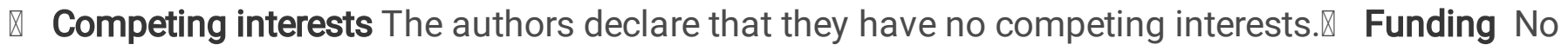
financial support for this study.

- Authors' contributions The author(Xiaojun Wang) is the inventor of the self-made single port device named Iconport. This article is the initial experience with Iconport. All authors participated in the 
study. All authors read and approved the final manuscript.

\ Acknowledgements The authors would like to acknowledge the leader of the Affiliated Hospital of Medical School of Ningbo University for supporting this study.

\section{Tables}

Table 1 Demographic including clinical characteristics of the study population

\begin{tabular}{|c|c|c|}
\hline Variables & value & $\mathrm{n}$ \\
\hline Mean age \pm SD (years) & $38.2 \pm 14.8 \square 12 \square 69 \square$ & 125 \\
\hline Gender & - & - \\
\hline Men & - & 77 \\
\hline Women & - & 48 \\
\hline BMI & I23.2 $\pm 3.5 \square 17.3-31.6 \square$ & 125 \\
\hline Postoperative diagnosis & - & 125 \\
\hline Acute simple appendicitis & - & 18 \\
\hline Chronic appendicitis & - & 5 \\
\hline Acute suppurative appendicitis & - & 87 \\
\hline Acute gangrenous appendicitis & - & 11 \\
\hline Periappendiceal abscess(Three months after conservative treatment) & - & 4 \\
\hline
\end{tabular}

BMI,body mass index

Table 2 Indication 
Inclusion/exclusion/ discharge criteria

\begin{tabular}{|c|c|c|}
\hline Inclusion & Exclusion & discharge \\
\hline Preoperative & Patients with clinical or radiological & The temperature was below 37 degrees \\
\hline diagnosis of & suspicion of appendicular pathology were & for more than 48 hours. \\
\hline appendicitis was & complicated by an abscess and/or local or & \\
\hline basically clear. & diffused peritonitis. & \\
\hline Stable vital signs. & The age below 12 years, pregnant patients, & Blood routine examination showed \\
\hline Cardiopulmonary & and patients who were unable to sign the & normal WBC and CRP was less than \\
\hline function can & informed consent form because of amental & 40mg/L. No redness, swelling and \\
\hline tolerate general & disorder were all excluded. & exudation was found in the incision. \\
\hline \multicolumn{3}{|l|}{ anesthesia } \\
\hline Laparoscopic & Patients with septic shock, patients with & Tolerance to oral diet. Ambulation. \\
\hline surgery was & contraindications for laparoscopic surgery & Absence of uncontrolled pain. \\
\hline acceptable & patients with contraindications for general & \\
\hline & anesthesia. & \\
\hline
\end{tabular}

Table 3 Perioperative data

\begin{tabular}{llc}
\hline Variables & Value & $\mathrm{n}$ \\
\hline Man operating time $\llbracket \mathrm{min} \square$ & $69.34 \pm 21.4(30-110)$ & 125 \\
Intraoperative blood loss $(\mathrm{ml})$ & $11.3 \pm 7.8(2-40)$ & 125 \\
Conversion rate & $4.8 \%(6 / 125)$ & 6 \\
Mean total hospital stay, day (range) & $5.0 \pm 1.8 \square 2-14 \square$ & 125 \\
VAS pain score(d1-d3 after surgery) & $1.8 \pm 0.7$ & 120 \\
\hline
\end{tabular}

Table 4 Perioperative complications 


\section{Perioperative complications}

\begin{tabular}{lll}
\hline Intraoperatve blood & $0 / 125$ & 0 \\
Wound infection & $0.04 \%[5 / 125 \square$ & 5 \\
Intraabdominal abscess & $0 / 125$ & 0 \\
Incisional hernia & $0 / 125$ & 0 \\
Postoperative ileus & $0 / 125$ & 0 \\
Postoperative bleeding & $0 / 125$ & 0 \\
Residual infection of the abdominal & $1.6 \%(2 / 125)$ & 2 \\
cavity was readmitted & & \\
\hline
\end{tabular}

\section{Figures}

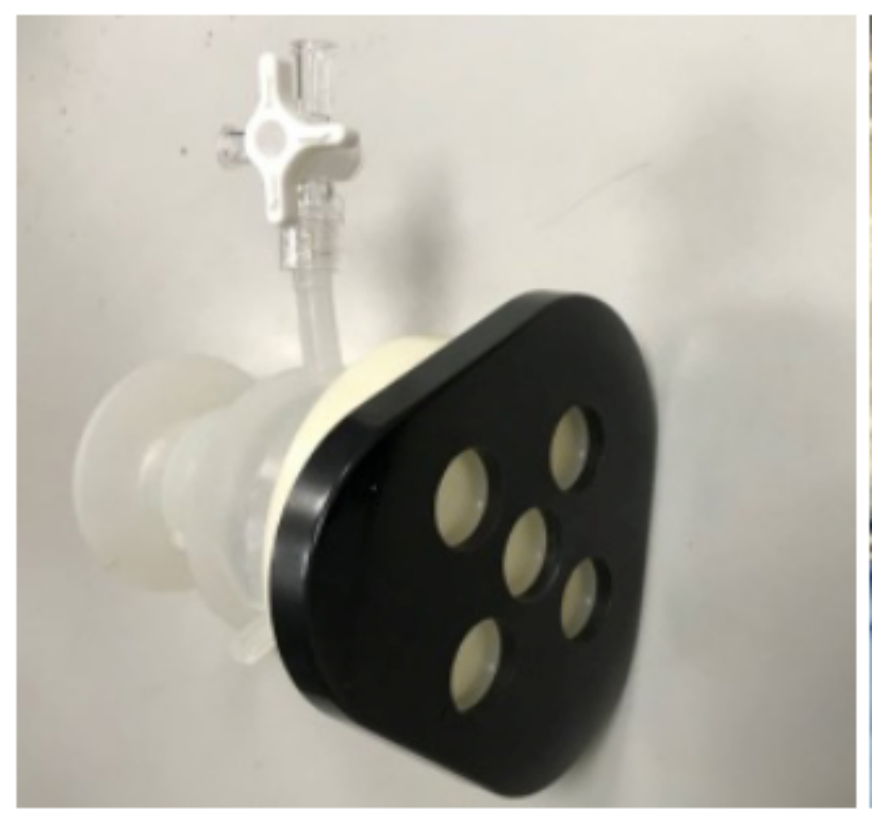

\section{Figure 1}

Single incision sealing device 


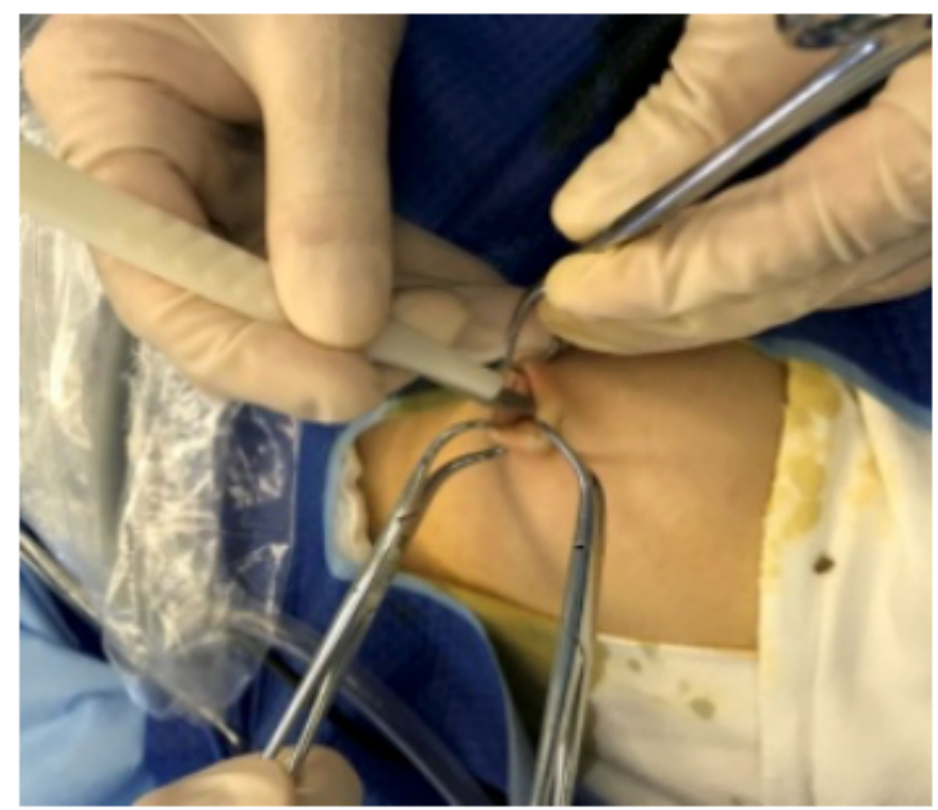

Figure 2

Umbilical incision

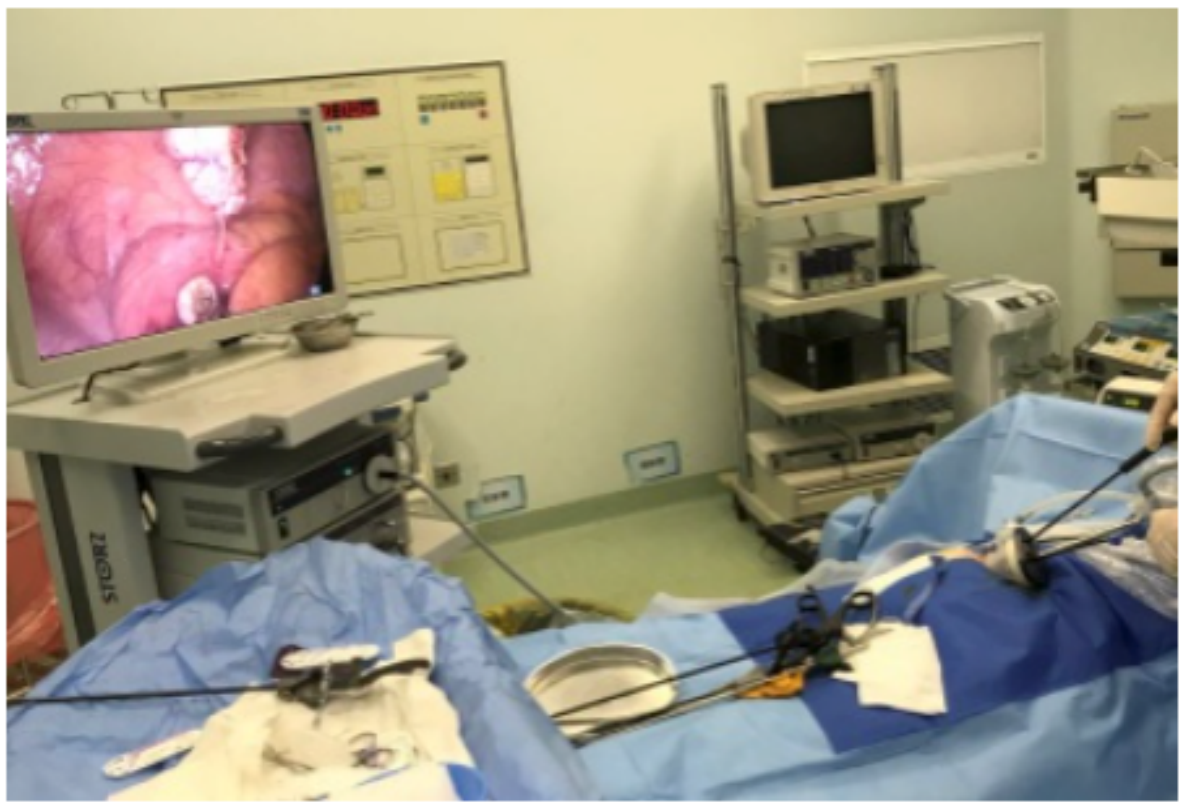

Figure 3

Laparoscopic appendectomy with conventional instruments 


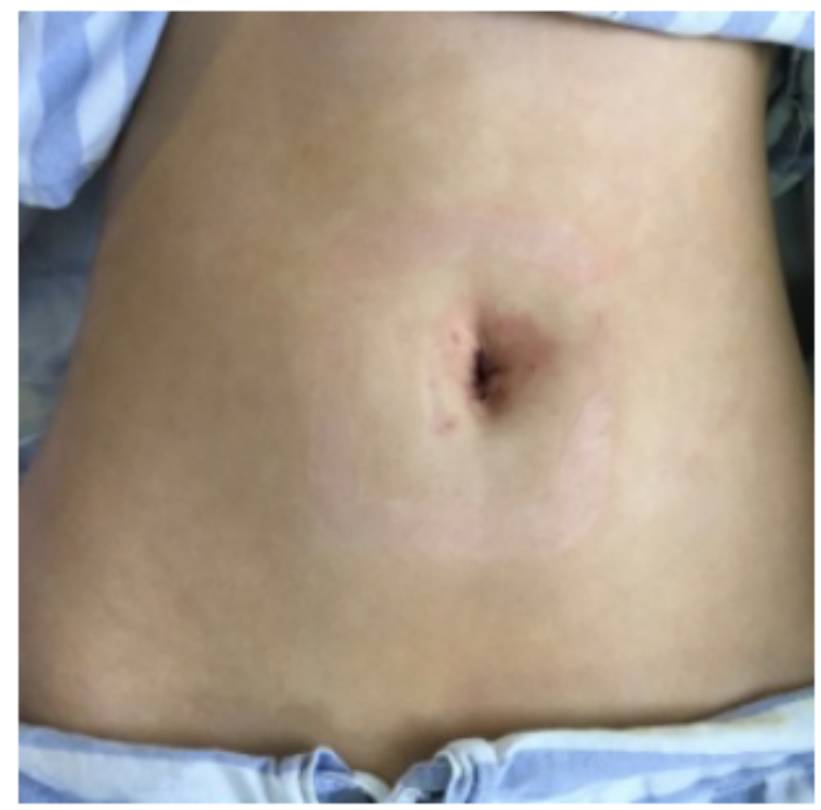

Figure 4

Postoperative umbilical incision marks 